\title{
Correction to: Bioavailability Enhancement of Olmesartan Medoxomil Using Hot-Melt Extrusion: In-Silico, In-Vitro, and In-Vivo Evaluation
}

\author{
Pankaj Jadhav, ${ }^{1}$ Vinod Gokarna, ${ }^{2}$ Vineeta Deshpande, ${ }^{3}$ and Pradeep Vavia ${ }^{1,4}$
}

Published online 2 October 2020

Correction to: AAPS PharmSciTech volume 21, Article number: 254 (2020). https://doi.org/10.1208/s12249-020-01780-3

During production, the supplemental file "Trajectory analysis of 5ns MD simulation of VA-64 and OLM.mpeg", as well as the "RunNo and Serial numbers", for Tables III and VII respectively, were inadvertently omitted from the published article (Tables I, II, III, IV, V, VI, and VII).

Publisher's Note Springer Nature remains neutral with regard to jurisdictional claims in published maps and institutional affiliations.

The online version of the original article can be found at https:// doi.org/10.1208/s12249-020-01780-3

\footnotetext{
${ }^{1}$ Department of Pharmaceutical Sciences and Technology, Institute of Chemical Technology, University under Section 3 of UGC Act 1956, Elite Status and Center of Excellence - Government of Maharashtra, TEQIP Phase II Funded, Mumbai, Matunga (E) 400019, India.

${ }^{2}$ Don Bosco Institute of Technology, Mumbai, Kurla (W) 400070, India.

${ }^{3}$ Department of Physics, Institute of Chemical Technology, University under Section 3 of UGC Act - 1956, Elite Status and Center of Excellence - Government of Maharashtra, TEQIP Phase II Funded, Mumbai, Matunga (E) 400019, India.

${ }^{4}$ To whom correspondence should be addressed. (e-mail: pr.vavia@ictmumbai.edu.in)
} 
Table I. Variables and Their Levels for Experimental Design

\begin{tabular}{|c|c|c|c|c|c|}
\hline \multirow[t]{2}{*}{ Variables } & \multicolumn{5}{|l|}{ Levels } \\
\hline & $-1.682(-\alpha)$ & -1 & 0 & 1 & $1.682(\alpha)$ \\
\hline \multicolumn{6}{|l|}{ Independent variables/factors } \\
\hline $\mathrm{X}_{1}$ : Drug concentration $(\% \mathrm{w} / \mathrm{w})$ & 6.59 & 10 & 15 & 20 & 23.41 \\
\hline $\mathrm{X}_{2}$ : Extrusion temperature $\left({ }^{\circ} \mathrm{C}\right)$ & 157 & 160 & 165 & 170 & 173 \\
\hline $\mathrm{X}_{3}:$ Screw speed (RPM) & 74.43 & 100 & 137.5 & 175 & 200.57 \\
\hline Dependent variable/response & Constrains & & & & \\
\hline Y: \% drug release in the first $10 \mathrm{~min}$ & Maximize & & & & \\
\hline
\end{tabular}

Table II. Solubility Parameter Values of Formulation Components and Miscibility Prediction

\begin{tabular}{llll}
\hline Compound & $\delta$ Solubility parameter & $\Delta \delta=\left(\left|\delta_{O L M}-\delta_{\text {Carrier }}\right|\right)$ & Miscibility \\
\hline OLM & 21.07 (MD simulation) & - & - \\
VA-64 & 20.53 (Piccinni et al., 2016) & 0.54 & Miscible \\
Soluplus & 19.90 (Piccinni et al., 2016) & 1.17 & Miscible \\
$\Delta \delta$ is difference in solubility parameters between drug and carrier & \\
\hline
\end{tabular}

Table III. Central Composite Design Run

\begin{tabular}{lllll}
\hline \multirow{2}{*}{ Run No } & \multicolumn{2}{l}{ Independent variables } & \multicolumn{2}{c}{ Dependent variable/response } \\
\cline { 2 - 5 } & $\mathrm{X}_{1}$-drug concentration $(\% \mathrm{w} / \mathrm{w})$ & $\mathrm{X}_{2}$-extrusion temperature $\left({ }^{\circ} \mathrm{C}\right)$ & $\mathrm{X}_{3}$-screw speed (RPM) & Y -\% drug release in the first 10 min \\
\hline 1 & 10 & 160 & 100 & 83.2 \\
2 & 20 & 160 & 100 & 74.2 \\
3 & 10 & 170 & 100 & 86.9 \\
4 & 20 & 170 & 100 & 66.5 \\
5 & 10 & 160 & 175 & 79.99 \\
6 & 20 & 160 & 175 & 61.9 \\
7 & 10 & 170 & 175 & 82.3 \\
8 & 20 & 170 & 175 & 74.3 \\
9 & 6.59 & 165 & 137.5 & 57.8 \\
10 & 23.41 & 165 & 137.5 & 94.4 \\
11 & 15 & 157 & 137.5 & 59.6 \\
12 & 15 & 173 & 137.5 & 93.01 \\
13 & 15 & 165 & 74.43 & 89.7 \\
14 & 15 & 165 & 200.57 & 83.5 \\
15 & 15 & 165 & 137.5 & 84.9 \\
16 & 15 & 165 & 137.5 & 84.1 \\
17 & 15 & 165 & 137.5 & 86.6 \\
18 & 15 & 165 & 137.5 & 82.8 \\
19 & 15 & 165 & 137.5 & 87.2 \\
20 & 15 & 165 & 137.5 & \\
\hline
\end{tabular}


Table IV. ANOVA for Response Surface Quadratic Model

\begin{tabular}{|c|c|c|c|c|c|c|}
\hline Source & Sum of squares & df & Mean square & $\mathrm{F}$ value & p-value Prob $>$ F & \\
\hline Model & 2867.08 & 9 & 318.56 & 6.61 & 0.0034 & Significant \\
\hline $\mathrm{X}_{1}$-drug concentration & 924.51 & 1 & 924.51 & 19.18 & 0.0014 & \\
\hline $\mathrm{X}_{2}$-temperature & 444.49 & 1 & 444.49 & 9.22 & 0.0125 & \\
\hline $\mathrm{X}_{3}$-screw speed & 168.48 & 1 & 168.48 & 3.50 & 0.0911 & \\
\hline $\mathrm{X}_{1} \mathrm{X}_{2}$ & 122.70 & 1 & 122.70 & 2.55 & 0.1417 & \\
\hline $\mathrm{X}_{1} \mathrm{X}_{3}$ & 89.04 & 1 & 89.04 & 1.85 & 0.2040 & \\
\hline $\mathrm{X}_{2} \mathrm{X}_{3}$ & 15.65 & 1 & 15.65 & 0.32 & 0.5814 & \\
\hline $\mathrm{X}_{1}^{2}$ & 887.82 & 1 & 887.82 & 18.42 & 0.0016 & \\
\hline $\mathrm{X}_{2}^{\frac{1}{2}}$ & 217.63 & 1 & 217.63 & 4.51 & 0.0595 & \\
\hline$X_{3}^{2}$ & 20.21 & 1 & 20.21 & 0.42 & 0.5318 & \\
\hline Residual & 482.01 & 10 & 48.20 & & & \\
\hline Lack of fit & 482.01 & 5 & 96.40 & & & \\
\hline Pure error & 0.000 & 5 & 0.000 & & & \\
\hline Cor total & 3349.09 & 19 & & & & \\
\hline
\end{tabular}

Table V. Percent Crystallinity Data

\begin{tabular}{llr}
\hline Sr. No. & Sample description & \% Crystallinity \\
\hline 1 & OLM & 52.72 \\
2 & VA-64 & 7.25 \\
3 & PM & 20.88 \\
4 & OLMEXT & 5.96 \\
\hline
\end{tabular}

Table VI. Pharmacokinetic Parameters

\begin{tabular}{lcc}
\hline Parameters & OLM & OLM EXT \\
\hline $\mathrm{C}_{\max }(\mu \mathrm{g} / \mathrm{mL})$ & 1.2396 & 3.3047 \\
$\mathrm{~T}_{\max }(\mathrm{h})$ & 2 & 0.5 \\
$\mathrm{AUC}_{0-24}(\mu \mathrm{g} / \mathrm{mL} * \mathrm{~h})$ & 7.2344 & 14.5842 \\
$\mathrm{t}_{1 / 2}(\mathrm{~h})$ & 8.65 & 5.05 \\
$\mathrm{~K}_{\mathrm{e}}\left(\mathrm{h}^{-1}\right)$ & 0.08018 & 0.1372 \\
$\mathrm{Cl}(\mathrm{mg}) /(\mu \mathrm{g} / \mathrm{ml}) / \mathrm{h}$ & 0.26956 & 0.15861 \\
$\mathrm{Vd}(\mathrm{mg}) /(\mu \mathrm{g} / \mathrm{ml})$ & 3.36205 & 1.156152 \\
$\mathrm{MRT}_{0-\infty}(\mathrm{h})$ & 13.9144 & 6.9345 \\
$\operatorname{Relative~bioavailability~}(\%)$ & 201.59 & \\
\hline
\end{tabular}

Table VII. Stability Evaluation of OLM EXT

\begin{tabular}{lllll}
\hline Sr. No. & Test & Stability period (months) & & \\
\cline { 3 - 4 } & & 0 & 3 & 6 \\
\hline 1 & Appearance & Transparent extrudes & Transparent extrudes & Transparent extrudes \\
2 & Assay & $99.17 \%$ & $98.87 \%$ & $97.78 \%$ \\
3 & $\%$ crystallinity & $6.01 \%$ & $5.98 \%$ & $6.05 \%$ \\
\hline
\end{tabular}

